\title{
Toward a Better Understanding of Non-Addicted, Methamphetamine- Using, Men who Have Sex with Men (MSM) in Atlanta
}

\author{
Brian J. Dew ${ }^{*}$
}

Georgia State University, Department of Counseling \& Psychological Services, Atlanta, GA 30302-3980, USA

\begin{abstract}
Methamphetamine use has increasingly become linked with sexual risk behaviors among men have sex with men (MSM). Yet, the majority of research has been done with methamphetamine dependent MSM or with samples in which addiction to the substance was not evaluated. Furthermore, research with methamphetamine-using MSM in the Southern U.S. is lacking. In this study, focus groups and in-depth interviews were conducted in order to understand the motives, context, and other facilitators and barriers of methamphetamine use among non-addicted MSM residing in Atlanta. Participants included 30 non-addicted, methamphetamine-using MSM and 16 local mental and public health officials. Findings from the first of this two-phase formative research project will result in the initial development of a community-tested, culturally-specific social marketing campaign and an individual-based intervention based in HIVtesting facilities.
\end{abstract}

Keywords: Methamphetamine, MSM, non-addicted users, social marketing campaign, drug use in Atlanta.

The use of methamphetamine has become increasingly prevalent throughout the United States in recent years, especially among men who have sex with men (MSM) living in urban settings $[1,2]$. Already recognized for its high propensity for addiction [3] and negative impact on mental $[4,5]$ and affective $[6,7]$ functioning, methamphetamine use is also correlated with significant public health concerns for MSM, specifically high-risk sexual behavior and HIV transmission. This link between HIV risk and methamphetamine use among MSM has been welldocumented in several large urban settings [8-12]. However, there has existed limited methamphetamine-related research conducted with MSM in Atlanta [13, 14]. This lack of investigation is surprising given that Atlanta possesses the highest rate of methamphetamine use among any major urban area east of the Mississippi River [15] and the largest proportion of MSM residents in the region [16]. Furthermore, HIV/AIDS infection rates in Atlanta rank in the top 10 of all metropolitan cities, with MSM comprising nearly half $(49 \%)$ of all cumulative AIDS cases and an increasing proportion of new HIV infections [17]. While recent epidemiological indicators suggest methamphetamine use in Atlanta is down since its peak in 2005 [15], it is difficult to determine the exact prevalence of and trends associated with methamphetamine use within the MSM community given the lack of empirical investigation.

Responses to the public health threats associated with methamphetamine use among MSM have mostly involved substance abuse treatment of dependent users and cityspecific, community-level education efforts. In the past decade, effective drug treatment programs have been

*Address correspondence to this author at the Department of Counseling \& Psychological Services; P.O. Box 3980, Atlanta GA 30302-3980, USA; Tel: 404 413-8168; Fax: 404 413-8013; E-mail: bdew@gsu.edu developed for methamphetamine-dependent MSM. These programs, although not available in the Southeast, have utilized contingency management strategies $[18,19]$, gayspecific cognitive-behavioral therapy [20, 21], gay-specific social support therapy [20], and combination psychopharmacology and cognitive-behavioral approaches [22]. It is unknown, however whether these treatment approaches yield the same results among MSM in other geographic regions. Large-scale, coordinated anti-methamphetamine campaigns have been implemented in metropolitan areas outside of the Southern U.S. [23, 24]. However, with the exception of Nanin and his colleagues' findings that public health antimethamphetamine initiatives in New York City were successful in reaching recent sexual at-risk MSM methamphetamine users [24], there has been minimal evaluation of these community campaigns to date.

The U.S. Centers for Disease Control and Prevention (CDC) hosted a national consultation in 2005 on the issue of methamphetamine use and sexual risk for HIV and other sexually-transmitted infections [25]. At this event, the state of the relevant science was summarized, ongoing programs were reviewed, and existing needs related to methamphetamine research and community programming were identified. Among the seven research suggestions recognized at this meeting were the following two:

R4. Explore the social and sexual context of methamphetamine use in populations for whom methamphetamine use is associated with sexual risk behavior.

R5. Develop and test prevention and risk-reduction interventions to decrease sexual risk behavior of nonaddicted methamphetamine users in populations for whom methamphetamine use is associated with sexual risk. 
Recent empirical efforts have examined select social and sexual contexts of methamphetamine use among MSM [12, 26-28]. Yet, there still exists a lack of research related to contextual factors that influence methamphetamine use, especially among MSM who do not currently meet the American Psychiatric Association's diagnostic criteria for methamphetamine dependence.

Although non-addicted men have been included in recent community-based research studies with MSM methamphetamine users $[2,11,29,30]$, few methamphetamine-related MSM studies have differentiated participants based on whether or not they met criteria for methamphetamine dependence. Consequently, there exists a void in the professional literature addressing the association of methamphetamine use and sex risk among MSM solely in the middle range of the spectrum between primary prevention and treatment for methamphetamine dependence. Thus, the aims of this two-phase formative research project were the following: (1) to investigate the contextual factors that influence methamphetamine use among non-addicted MSM residing in a region that has been largely ignored in the professional literature; (2) to develop preliminary social marketing concepts and intervention methods and materials based on research findings and on psychosocial theory; (3) to employ a community-based evaluation of initial social marketing concepts and intervention methods and materials; and (4) to revise and further develop social marketing concepts and intervention components addressing nonaddicted methamphetamine use among MSM.

\section{METHODS}

\section{Study Design}

This two-phase formative research was a collaborative project funded through a seed grant between the CDC and Georgia State University. Phase 1 consisted of three focus groups with non-addicted methamphetamine-using MSM and one focus group each with Atlanta-based community mental health providers and AIDS risk-reduction specialists. Individual interviews with 10 non-addicted methamphetamine using MSM were also conducted in order to confirm and/or to expand upon focus group results.

The purpose of Phase 2, which is ongoing, is to utilize the findings from Phase 1 to: 1) further develop methamphetamine-related social marketing campaigns that target MSM in Atlanta; 2) obtain feedback on methamphetaminerelated social marketing concepts from local drug-using MSM and HIV testing officials to develop further the social marketing campaigns; and 3) obtain feedback on proposed intervention components from drug-using MSM and local HIV testing officials. Only the results from Phase 1 are presented in this paper.

\section{Participants and Procedure}

A targeted strategy comprised of active and passive techniques was used in the recruitment of non-addicted, methamphetamine-using participants. Active recruitment consisted of street outreach and recruitment at MSMoriented bars, dance clubs, and bath houses identified by methamphetamine users as popular venues. Recruitment staff actively approached individuals when they entered the venue, explained the purpose of the study, and offered an invitation to complete a 3 to 5 -minute screening, including a section used to determine non-addicted use of methamphetamine. Individuals answered questions that corresponded to the seven American Psychiatric Association's Diagnostic and Statistical Manual for Mental Disorders (DSM) IV-TR criteria for dependence [31]. Sample screening questions included, "In the last 12 months, have you had a persistent desire or unsuccessful efforts to cut down or control your methamphetamine use?" and "Have you given up or reduced important social, occupational, or recreational activities because of your methamphetamine use in the past 12 months?" If the person answered yes to three or more dependence-related questions, he was determined to be addicted and therefore not eligible to participate. Mental health referrals, including local substance abuse treatment programs and psychotherapists, were provided to individuals found to be methamphetamine dependent. If eligible and willing to participate, individuals were either scheduled for an appointment or asked to call the project telephone number to arrange an appointment.

Passive recruitment of non-addicted methamphetamine users included advertisements in local MSM-oriented newspapers as well as distribution of study materials in MSM social venues (e.g. local bars, dance clubs, sex clubs, and bath houses) and in AIDS service organizations. These efforts involved the distribution of recruitment cards that provided information about the study as well as contact information for the project's research office. Interested persons were encouraged to contact the research office either via phone or email. Participant screening resulting from passive recruitment efforts occurred solely on the telephone. The recruitment of HIV risk reduction experts (AIDS service organization staff and public health department officials) and community mental health professionals (substance abuse treatment officials, clinical psychologists, licensed professional counselors, and clergy) was conducted via phone calls and emails. These individuals were identified as a result of this author's existing community relationships and by colleague referrals. The study protocol was approved by the Institutional Review Boards of Georgia State University and the CDC. All data were collected between October 2008 and February 2009.

Inclusion criteria for non-addicted MSM users of methamphetamine included: 1) be 18 years of age or older; 2) self-identify as male; 3) understand and read English; 4) live within the geographic metropolitan Atlanta area; 5) report methamphetamine use at least one time in the past three months; 6) self-identify as a man who had oral or anal sex with a man at least once in the last three months; and 7) presently does not meet criteria for methamphetamine dependence based on criteria identified by the DSM-IV TR [31]. Inclusion criteria for the community officials included offering professional services to MSM within the Atlanta metropolitan area, having knowledge of methamphetamine use and its impact within Atlanta's MSM community, and being at least 18 years old. 
A semi-structured format was used for the focus groups in order to illicit exploratory responses to pre-established questions, while providing enough flexibility for participants to identify influences on their methamphetamine use as well as provide insight into potential educational campaigns and interventions. Examples of focus group questions included "What is it about using meth that is appealing to MSM in Atlanta, compared to use of other substances?" and "What do you think could be done to change a possible link between meth use and sex among non-meth addicted MSM in Atlanta? Semi-structured individual interviews were also used to probe in greater detail findings from previous focus groups. An example of an individual question included, "What could be done on an individual as well as a community-level to change the link between methamphetamine use and HIV."

\section{Data Analysis}

Phase 1 consisted of 5 focus groups ( 3 comprised of nondependent methamphetamine-using MSM and 2 of public and mental health professionals) and 10 in-depth individual interviews with non-addicted methamphetamine-using MSM. All focus groups and individual interviews were audio taped and transcribed. All focus groups were led by the same two co-facilitators, while two observers completed field notes [32] which included descriptive observations of participant appearance, behavior, affect, and interaction. Notes also were taken describing the interview location and setting, the co-facilitators' inferences and emotional reactions during the interaction, and any unusual circumstances that may have influenced data content or collection [32].

Data analysis followed an established inductive process of item, pattern, and structural analysis [32, 33]. Inductive analysis allowed patterns to emerge through a detailed review of data and also provided a method of accounting for researcher-participant interactions. A team of two coders independently reviewed a data set (transcriptions and field notes) and compiled their own list of codes. Members of this dyad then met to compare and discuss findings, and to develop a comprehensive coding list for that transcript. Items were kept as close as possible to specific and concrete from transcripts in order to reduce interpretive drift [33]. Throughout item-level analysis the research team identified patterns by item frequency, similarities, omissions, or by participant identification of patterns. Finally, the research team looked for emergent relationships between patterns (themes) that pertained to the research questions.. After coding three transcripts separately, a minimum interrater agreement standard of $90 \%$ was achieved [34] thereby allowing the two coders to review independently the remaining transcripts.

\section{RESULTS}

A total of 78 potential methamphetamine-using MSM participants were screened either in the field $(n=28)$ or by telephone $(n=50)$. Nearly $44 \%$ of these interested persons were determined to be ineligible either because they met the criteria for methamphetamine dependence $(n=22)$ or they had not used methamphetamine in the previous three months $(n=12)$. A total of 20 non-addicted methamphetamine-using MSM participated in one of three 90-minute focus groups, while 10 non-addicted methamphetamine-using MSM completed in-depth individual interviews lasting approximately 60-90 minutes. The mean age of methamphetamineusing participants was 40.2 years (range: 24-56) and the racial composition was evenly split (48\% each) between African American and White participants. Focus groups consisting of public $(n=6)$ and mental health professionals $(n=10)$, were less racially-mixed (63\% White, 25\% AfricanAmerican, and 12\% Hispanic) (age range: 26 to 62 years old). Specific themes evolved from analyzing content related to the following three areas of inquiry: (1) motivators for non-addictive methamphetamine use; (2) facilitators to avoid dependent use of the drug; and (3) assessment of need for local education campaigns and intervention efforts.

\section{Motivators Impacting Recreational Use}

Three themes were found to have motivated non-addicted MSM use of methamphetamine. First, focus group and individual interview participants cited the drug's ability to enhance sexual pleasure as an important motivator to use. The drug's ability to increase sexual arousal and stamina and enhance one's ability to achieve an erection were most often cited as physiological changes associated with methamphetamine use. These sexual side effects were often combined with memory suppression, thereby diminishing potentially negative affective responses such as guilt and shame during male-to-male sex. This combination of enhanced physiological response and minimized negative mood led to lowered sexual inhibitions and amplified selfconfidence during sex. This alleviation of internalized homonegativity was especially relevant to MSM participants raised in the Southern U.S. or in conservatively religious homes outside the region. A 28 year-old, White, HIVnegative individual interview participant noted:

It really made me less inhibited...gave me more stamina, more energy. It was like taking Viagra almost but you don't have to go through a doctor to get it. Sex is also better with meth because of the shame, it kinda erases the shame that you are doing something that is not acceptable. Taboo. It kinda erases that almost totally, For me it erased that line.

A second motivator to use methamphetamine recreationally was the drug's perceived "cost effectiveness" compared to other substances. Whereas participants had once viewed methamphetamine as an expensive drug to be "used on special occasions," decreases in local retail pricing resulted in greater affordability. This appeal of lowered prices was often combined with the recognition of the drug's extended high compared to other drugs. White users were more likely to prefer methamphetamine over Gamma hydroxybutyrate (GHB), marijuana, and powder cocaine in a cost/reward comparison. African American participants tended to view methamphetamine favorably when compared to crack cocaine, especially during sexual encounters. Both White and non-White users of the drug viewed the supply 
and use of methamphetamine as increasing in Atlanta's comparatively large African American MSM community. A 38 year-old, HIV-positive, African American methamphetamine user described the drug's appeal compared to crack cocaine:

I like the longevity of it [methamphetamine]. It's a lot cheaper per use than crack cocaine. It's cheaper in the long run than buying rocks. You don't have to keep going out, you're not worrying about going to get some more because you have enough to get you through and it lasts longer than crack.

In addition to being sexually gratifying and cost-effective, methamphetamine users and mental health professionals cited escapism as a motivator to use methamphetamine. Escapism from one's financial worries, relationship difficulties, physical health issues, and depression were identified as motivators to use the drug. A 35 year-old, HIV-negative, White recreational user of methamphetamine described why he uses:

I mean it sucks right now. The economy is screwed up and no one is hiring and I don't know when that will change. Yeah....sometimes I use [meth] to escape from the world around me but we gay men have been using drugs for a long time to do just that, escape. With meth, you just forget your problems a little longer than with other drugs.

For participants living with HIV/AIDS, this desire to escape was even more exacerbated. HIV-positive, methamphetamine-using participants explained the allure of the drug as a means to cope in the following three ways: (1) temporarily causes the user to forget the reality of being HIV-positive and the societal stigmatization attached to the disease; (2) minimizes the risk of rejection from sexual partners who are also using methamphetamine; and (3) lowers the expectation of having to disclose one's HIV status to potential sexual partners. Two local mental health professionals shared the following exchange in a focus group:

Participant A: For my HIV-positive clients, the use of meth tends to... help a person cope with something a person doesn't really want to deal with.

Participant B: What I have noticed is that it [meth use] seems to be a numbing out process and a coping mechanism for a lot of my HIVpositive gay male clients. Yet, it makes what you're going through worse.

\section{Facilitators to Avoid Heavier Methamphetamine Use}

Even though participants acknowledged specific motivators to using methamphetamine recreatinally, there existed factors that impeded an individual's potential trajectory to addicted use. First, participants cited being pleased with their current life functioning, while fearing negative physical, occupational, and social consequences that would result from more frequent use. A 38 year-old, HIV-positive, White methamphetamine user said:

Everyone has their own particular reasons not [to use more]. My choice of not doing meth on a regular basis is because I like my life the way it is and I would not be able to function the way my life should be if I was doing [meth] all the time.

In addition to their current life satisfaction, methamphetamine-using participants acknowledged that knowing at least one methamphetamine addict was instrumental in their decision to not using the drug more frequently. Observation of others' addictive behavior, including compulsive sexual "acting out" and paranoia, as well as recognition of more subtle effects of frequent use such as changing social networks and increased isolation, were acknowledged by MSM users of the drug as well as mental health professionals. Witnessing the impact of dependent use on one's physical health also served as a powerful facilitator to avoid heavier use. A 43 year-old, White, HIV-positive methamphetamine user related:

It takes a toll on your face. Can you imagine what your insides look like? And your teeth, I don't ever want to get to that point. I was doing this drug because I wanted someone, because I wanted my inhibitions to be less when having sex and then that very same drug could cause someone not want to come anywhere near me because my face or my mouth or my teeth are all messed up because of me using the very same drug that I used a year earlier to be this sexual powerhouse. I think that is such a scary irony.

Relationships with methamphetamine dependents also reinforced the perception of the drug's addictiveness compared to other substances. As a result, certain harm reduction approaches to using methamphetamine were used in order to avoid becoming addicted, including the choice not to smoke or inject the drug, reduce the number of "using days" to just weekends, or refusing to ever buy the drug, instead relying on others to provide it. A 24 year-old, HIVnegative, White MSM stated:

One of the reasons I never want to use meth really heavy is that you can get real addicted to it. I mean I have friends who went from trying it to getting hooked in no time, it was less than a month and they were using every day. that is why I don't smoke it or slam it. I mean it's more addictive than any drug I have put into my body and I have tried many of them.

A third facilitator to avoid heavier methamphetamine use involved the drug's association with HIV and its deleterious impact on the HIV-positive individual. MSM users of methamphetamine and public and mental health officials were unanimous in their awareness of the link between methamphetamine use and HIV sexual risk behavior. Although not identified by the "professional" focus group 
participants, users of the drug identified the fear of contracting HIV as a factor in regulating their methamphetamine use of the drug. Participants acknowledged that methamphetamine addiction could lead to out-of-control sexual behavior that could significantly increase the likelihood of becoming HIV-positive.

An important element of the perceived connection between HIV and methamphetamine use was the inconsistent self-disclosure of one's HIV-positive status prior to sex for persons using methamphetamine. Two HIV-positive participants in one MSM focus group shared the following exchange:

Participant A: It doesn't matter how high I am it's that constant in the background. I don't play with people who aren't positive. I've been high to the point where my eyeballs pop, and I was still not going to have sex with anyone who is negative. It's in the back of my mind. It's always there. I'm pos what about you? I don't think there's any excuse for anyone whose pos to have sex with anyone whose negative. I would rather jerkoff, kick them out, anything. I've actually had people lie to me twice and meth was involved in both situations.

Participant B: In my experience, I've never had anyone tell me that they were positive. All the guys in the party nobody ever say it, they just want the pipe... But no one has ever come out and said before I hit this meth I'm HIVpositive. Because basically not all guys are like you [participant A].

Participant A: I understand that I mean I inject meth - but no matter how fucked up I am I realize that if someone's negative and are coming to me and saying they want my pos load... first it turns me off and second of all, I still have that conscious in my head oh I'm not going to pass that on.

Participant B: Well, you're one in a million.

HIV-positive participants recognized that addicted use of the drug would have extremely negative impact on their physical well-being. HIV-positive participants feared that if they ever became addicted to the drug, they would fail to adhere to their anti-retroviral medication schedule and therefore potentially build up a resistance to their drug regimen. Public and mental health professionals cited numerous cases in which their methamphetamine-using HIV-positive MSM clients would skip appointments, fail to take their medication consistently, and report higher viral load counts and impaired immune functioning. These community-based professionals witnessed these events in both addicted, as well as non-addicted methamphetamine users.

The final theme cited as a catalyst to limit the use of methamphetamine involved the viewing of billboards and print advertisements based on a series of fear messages conducted in 2006-2007. Sponsored by a local network of concerned citizens, these individuals placed a one-month billboard ("Meth = Death") in the heart of Atlanta's MSM community, as well as included advertisements in MSMoriented newspapers and magazines that demonstrated the negative effects that the drug can have on the body. While methamphetamine users found these ads to be noneducational ("What do these ads tell me? Does it show me how to use more safely? Does it tell me where I can get help?") and unrealistic ("I am a meth user and I haven't died nor have my friends"), participants cited their effectiveness in raising awareness of methamphetamine's negative effects. These advertisements were cited as helpful because participants recognized them as the only community response to the methamphetamine problem within Atlanta's MSM community.

\section{Assessment of Need for Local Education Campaigns and Intervention Efforts}

Participants noted the lack of a coordinated community and individual-level public health response, including prevention and interventions tailored to meet the local community, as "troublesome" and "shameful." The development, implementation, and evaluation of an Atlanta-based social marketing campaign were viewed by nearly all participants as needing to occur immediately. This campaign would ideally be conducted both online with select MSMoriented websites and in print advertisements placed in particular bars, clubs, and sex clubs. Furthermore, MSM methamphetamine-using participants and public and mental health professionals provided ideas for individual interventions that could be offered by existing MSM-related service organizations, including specific HIV testing facilities.

\section{DISCUSSION}

Although multiple cities outside the South have developed social marketing campaigns targeting the reduction of methamphetamine use among MSM, it is uncertain if these concepts, if replicated, would be the most advantageous for Atlanta's MSM community. Rather, results from this study suggest that understanding local contextual influences on the drug's use by MSM is critical in the development of effective outreach efforts. First, results from this study indicate that any social marketing campaign and intervention efforts conducted in Atlanta's MSM community must appeal to diverse racial audiences, especially African American MSM, and varying age groups. This finding is not consistent with local drug indicators, including metropolitan Atlanta public substance abuse treatment and prison admissions, which indicate that persons admitted due to methamphetamine are over 95\% White [13]. The range and mean in MSM participant ages indicate that methamphetamine is not limited to young adult users, and therefore, any integrated community education and intervention effort should include recruitment strategies, including the use of images, that reflect this diversity in age.

In addition to being diverse in its appeal, findings from this study support the need for the creation, implementation, 
and evaluation of Atlanta-based social marketing campaigns and intervention efforts that consider the cultural impact of living in the most socially conservative region of the country. Despite currently residing in a large urban setting, participants reared in the South were likely to report internalization of negative messages related to being nonheterosexual, many of which were religiously-based. Public and mental health professionals could enhance service delivery with non-addicted methamphetamine users by integrating approaches that target the reduction of drug use, but also addressing internalized homophobia and other underlying issues that potentially could influence methamphetime use prevalence.

Although previous fear-based, MSM-focused advertising heightened awareness of the physical risk associated with methamphetamine use, these efforts provided negligible impact on educating the community about the associations between HIV and the drug, encouraging effective harm reduction strategies related to using more safely, and giving resources for obtaining more information. By focusing solely on "meth = death" and other fear-based messages, education efforts neglected the motivators to use the drug (e.g. sexual, cost effective, and escapism), which potentially increases its stigmatization and enhances judgmental attitudes toward its users.

Findings from Phase 1 will assist in the development of 4-5 social marketing concepts developed specifically to target local non-dependent users of methamphetamine. Once the social marketing ideas are developed, street interception with over 200 gay and bisexual-identified participants during MSM-oriented venues such as festivals, bars, and clubs will be conducted in order to receive feedback on the effectiveness of each social marketing concept. Further community-based evaluation of the various social marketing campaign concepts will be conducted with 10 local recreational methamphetamine-using MSM who will participate in individual interviews as well as approximately 20 methamphetamine-using MSM who will participate in one of three focus groups. These efforts will result in the selection of two social marketing campaign concepts identified via community-based testing to be most effective in reaching recreational methamphetamine-using MSM in Atlanta.

Besides identifying important elements of the social marketing campaign, results from our formative research found that an individual intervention based in HIV testing facilities could be effective in providing education to recreational methamphetamine-using MSM. Although the feedback obtained in Phase 1 did not provide specifics related to the exact nature of this individual intervention, methamphetamine-using and public and mental health professionals acknowledged that integrating a methamphetamine intervention with a MSM's HIV test could be timely, efficient, and beneficial in producing sexual and other at-risk behavioral changes. Further refinement of such an intervention will be aided by individual interviews and a focus group with both HIV testing officials and recreational methamphetamine-using MSM to be held during Phase 2.
Information related to the HIV testing process, attitudes towards participating in a drug-related intervention during a potentially anxiety-heightened event, incentives for participating, methods of delivering the intervention (e.g., computer versus in-person) and resources to conduct such an intervention will be collected.

There are several limitations that must be acknowledged when discussing the outcomes of this study. First, this formative research's small sample size may not reflect the entire population of non-addicted methamphetamine users in Atlanta. Compared to other cities with large MSM communities, few research studies related to drug use and sexual behaviors involving gay and bisexual men in Atlanta have been conducted. Due to the combination of the stigma attached to drug use and the cultural conservatism pervasive in the Southern U.S., it is possible that the men who agreed to participate in Phase 1 of this study were different than the larger MSM non-addicted methamphetamine-using population.

While members of the research team were trained to screen for dependent use, it is possible that the DSM IVTR's criteria for addiction to methamphetamine did not adequately assess whether the participant was a non-addicted user of the drug. Consequently, it is possible that individuals who were actually addicted to the substance were incorrectly identified as non-dependent and included in this study. Efforts were made to reduce the chance of this error occurring as all research team members re-screened participants prior to the individual interview or focus group. Finally, as with all self-repot based studies, it is possible that participants answered questions or interacted in ways that made themselves look more socially acceptable to the interviewer, group facilitator, and/or focus group members. Although participants were encouraged to share openly their thoughts, behaviors, and perspectives and speak in "ordinary, everyday language," it is logical to assume that participants were reluctant to share particular sexual behaviors while high or share complete histories of their drug use.

\section{CONCLUSION}

Information obtained from Phase 1 of this formative study can assist in the development of culturally appropriate, effective social marketing campaign and intervention efforts that target non-addicted, methamphetamine-using MSM in Atlanta. The research study's Phase 2 will allow for the further development and refinement of these educational and intervention endeavors. While it unknown whether or not to expect the same community needs in regions outside of the Southern U.S., or even in other metropolitan areas within the South, steps taken to develop a methamphetamine-related campaign for MSM could potentially be beneficial to community health providers elsewhere.

\section{ACKNOWLEDGEMENTS}

The author acknowledges the contributions of Dr. Gordon Mansergh for his valuable contribution to this research. In addition, I give special thanks to Mistie Storie, Josh Castleberry, Marc Pimsler, and Jennifer Smith, team members of Project MAP, for their efforts in recruitment, data collection, and qualitative analysis during Phase 1. 


\section{REFERENCES}

[1] Halkitis PN, Solomon, TM, Moeller RW, et al. Methamphetamine use among gay, bisexual and non-identified men-who-have-sexwith-men: An analysis of daily patterns. J Health Psychol 2009; 14: 222-31.

[2] Parsons JT, Bimbi DS. Intentional unprotected anal intercourse among men who have sex with men: Barebacking-from behavior to identity. AIDS Behav 2007; 11: 277-87.

[3] Castro FG, Barrington EH, Walton MA, Rawson RA. Cocaine and methamphetamine: Differential addiction rates. Psychol Addict Behav 2000; 14: 390-6.

[4] Moone M, Do KS, Park J, Daijin K. Memory impairment in methamphetamine dependent patients. Int J Neurosci 2007; 117: 19.

[5] Rendell PG, Mazur M, Henry JD. Prospective memory impairment in former users of methamphetamine. Psychopharmacology 2009; 203: 609-16.

[6] Looby A, Earleywine M. The impact of methamphetamine use on subjective well-being in an Internet survey: Preliminary findings. Hum Psychopharmacol 2007; 22: 167-72.

[7] Semple SJ, Zians J, Strathdee SA, Patterson TL. Psychosocial and behavioral correlates of depressed mood among female methamphetamine users. J Psychoactive Drugs Sarc Suppl 2007; 4: 353-66.

[8] Fernandez MI, Varga LM, Perrino $\mathrm{T}$, et al. The Internet as recruitment tool for HIV studies: viable strategy for recruitment atrisk Hispanic MSM in Miami? AIDS Care 2004; 16: 953-63.

[9] Halkitis PN, Parsons JT, Wilton L. An exploratory study of contextual and situational factors related to methamphetamine use among gay and bisexual men in New York City. J Drug Iss 2003; 33: 413-32.

[10] Taylor MM, Aynalem G, Smith LV, Montoya J, Kerndt P. Methamphetamine use and sexual risk behaviors among men who have had sex with men diagnosed with early syphilis in Los Angeles County. Int J STD AIDS 2007; 18: 93-7.

[11] Semple SJ, Zians J, Grant I, Patterson TL. Sexual risk behavior of HIV-positive methamphetamine-using men who have sex with men: The role of partner serostatus and partner type. Arch Sexual Behav 2006; 35: 461-71.

[12] Kurtz S. Post-circuit blues: motivations and consequences of crystal methamphetamine use among gay men in Miami. AIDS Behav 2005; 9: 63-72.

[13] Benotsch EG, Kalichman S, Cage M. Men who have met sex partners via the Internet: Prevalence, predictors, and implications for HIV prevention. Arch Sex Behavior 2002; 31: 177-83.

[14] Dew BJ, Elifson K, Sterk C. Difference in HIV sexual risk behaviors between heterosexual and non-heterosexual male users of methamphetamine. J Drug Issues 2007; 37: 281-98.

[15] Dew BJ. Patterns and trends of drug use in Atlanta. In National Institute of Drug Abuse's Epidemiologic Trends in Drug Abuse. Bethesda, MD: US Department of Health and Human Services 2008.

[16] Lee R. Atlanta ranks third in percent of gays: Analysis finds gay couples up nationwide 30\% since 2000. [accessed: 2006 Nov 24]. Available from: http://www.sovo.com/2006/11-24/news/localnews/ rise.cfms

[17] Center for Disease Control and Prevention. Reported AIDS cases and annual rates (per 100,000 population), by metropolitan statistical area of residence, 2005, 2006, and cumulative-United States and Puerto Rico. National Center for HIV, STD and TB
Prevention, Centers for Disease Control and Prevention, Department of Health and Human Services 2007.

[18] Shoptaw S, Reback C, Peck J, et al. Behavioral treatment approaches for methamphetamine dependence behaviors and HIVrelated sexual risk among urban gay and bisexual men. Drug Alcohol Depend 2005; 78: 125-34.

[19] Strona FV, McCright J, Hjord H, et al. The acceptability and feasibility of the positive reinforcement opportunity project, a Community-based contingency management methamphetamine treatment program for gay and bisexual men in San Francisco. J Psychoactive Drugs SARC Suppl 2006; 3: 377-81.

[20] Shoptaw S, Reback C, Larkins S, et al. Outcomes using two tailored behavioral treatments for substance abuse in urban gay and bisexual men. J Subst Abuse Treat 2008; 35: 285-93.

[21] Shoptaw S, Reback C, Peck J, et al. Behavioral treatment approaches for methamphetamine dependence and HIV-related sexual risk behaviors among urban gay and bisexual men. Drug Alcohol Depend 2005; 78: 125-34.

[22] McElhiney MC, Rabkin JG, Rabkin R, Nunes EV. Provigil (modafinil) plus cognitive behavioral therapy for methamphetamine use in HIV+ gay men: A pilot study. Am J Drug Alcohol Abuse 2009; 35: 34-7.

[23] Michaels M. Groups tailor their anti-methamphetamine messages to reach potential addicts. Chron Philanthropy 2007; 12.

[24] Nanin JE, Parsons JT, Bimbi DS, Grov C, Brown JT. Community reactions to campaigns addressing crystal methamphetamine use among gay and bisexual men in New York City. J Drug Educ 2006; 36: 297-315.

[25] Mansergh, G, Purcell DW, Stall R, et al. CDC consultation on methamphetamine use and sexual risk behavior for HIV/STD infection: summary and suggestions. Public Health Rep 2006; 121 : 127-32.

[26] Parsons JT, Kelly BC, Weiser JD. Initiation into methamphetamine use for young and bisexual men. Drug Alcohol Depend 2007; 90: 135-44.

[27] Halkitis PN, Palamar JJ. GHB use among gay and bisexual men. Addict Behav 2006; 31: 2135-9.

[28] Green AI, Halkitis P. Crystal methamphetamine and sexual sociality in an urban gay subculture: An elective affinity. Cult Health Sex 2006; 8: 317-33.

[29] Wilton L, Halkitis P, English G, Roberson M. An exploratory study of barebacking, club drug use, and meanings of sex in Black and Latino gay and bisexual men in the age of AIDS. J Gay Lesbian Psychother 2005; 9: 49-72.

[30] Mausbach BT, Semple SJ, Strathdee SA, Zians J, Patterson TL. Efficacy of a behavioral intervention for increasing safer sex behaviors in HIV-positive MSM methamphetamine users: results from the EDGE study. Drug Alcohol Depend 2007; 87(2-3): 24957.

[31] American Psychiatric Association. Diagnostic and statistical manual of mental disorders: DSM IV-TR. $4^{\text {th }}$ ed. Washington DC 2000 .

[32] LeCompte MD, Schensul JJ. Designing and conducting ethnographic research (Ethnographer's toolkit, Book 1). Walnut Creek, CA: Altamira 1999.

[33] Lincoln YS, Guba EG. Naturalistic inquiry. New Park, CA: Sage 1985.

[34] Bakerman R, Gottman JM. Observing interaction: An introduction to sequential analysis. $2^{\text {nd }}$ ed. Cambridge: Cambridge University Press 1997.

(C) Brian J. Dew; Licensee Bentham Open.

This is an open access article licensed under the terms of the Creative Commons Attribution Non-Commercial License (http: //creativecommons.org/licenses/by-nc/ $3.0 /$ ) which permits unrestricted, non-commercial use, distribution and reproduction in any medium, provided the work is properly cited. 